\title{
Postprandial cardiogenic syncope caused by gastric polyp-induced pyloric obstruction in an elderly woman with a giant hiatal hernia: a case report
}

\author{
Hideyuki Saito ${ }^{1}$, Tatsuya Miyazaki ${ }^{{ }^{*}}$ (D, Makoto Sohda', Makoto Sakai ${ }^{1}$, Hiroaki Honjyo ${ }^{1}$, Yuuji Kumakura', \\ Tomonori Yoshida', Takehiko Yokobori ${ }^{1,2}$, Koji Kurosawa ${ }^{3}$ and Hiroyuki Kuwano ${ }^{1}$
}

\begin{abstract}
Background: Hiatal hernias are common. In some reports, hiatal hernias have been implicated in causing dyspnea, syncope, and heart failure.

Case presentation: An 82-year-old woman with a hiatal hernia was admitted to our hospital because she had experienced postprandial syncope during the last few years. Esophagogastroduodenoscopy revealed a large hiatal hernia and a pedunculated polyp of the stomach antrum that fit into the pylorus. An upper gastrointestinal contrast study showed that the entire stomach had relocated to the thoracic cavity and that the body of the stomach was located above the fundus, resulting in a so-called upside-down stomach. Contrast-enhanced computed tomography revealed that a large portion of the stomach, transverse colon, and part of the pancreas were present in the mediastinum. We then performed transthoracic echocardiography followed by a water pouring test using a nasogastric tube. After instillation of $2000 \mathrm{ml}$ of saline, the left atrium was markedly compressed and the area of the mitral annulus was reduced. We determined that stomach dilation by the hiatal hernia and gastric polyp had caused the syncope. The patient underwent laparoscopic hiatal hernia repair and endoscopic gastric polypectomy, and she experienced no syncopal episodes for 5 months postoperatively. Conclusions: Clinicians should recognize that a large hiatal hernia may be a risk factor for syncope.
\end{abstract}

Keywords: Hyperplastic polyp, Upside-down stomach, Laparoscopic hiatal hernia repair, Endoscopic polypectomy

\section{Background}

Hiatal hernias in adults often develop in elderly people, and the main symptoms are heartburn, chest pain, reflux, and dysphagia. Some reports have described cardiac complications such as arrhythmia and heart failure caused by giant hiatal hernias [1, 2]. However, few reports have mentioned heart failure-induced syncope in patients with giant hiatal hernias [3-10]. Reports of such cases are very important to prevent oversight in primary care. No reports have described a syncopal episode caused by a hiatal hernia and prolapsing gastric polyp. We herein document

\footnotetext{
*Correspondence: tatsuyamiyazaki@gunma-u.ac.jp

'Department of General Surgical Science, Gunma University Graduate School of Medicine, 3-39-22 Showa-machi, Maebashi, Gunma 371-8511, Japan Full list of author information is available at the end of the article
}

such a case in a patient with a giant hiatal hernia whose chief complaint was loss of consciousness.

\section{Case presentation}

An 82-year-old woman with a hiatal hernia was admitted to our hospital for a detailed examination. She reported repeated postprandial syncope once a year, and the frequency had increased in recent years. She had undergone close examination in other hospitals at the time of the syncopal episodes, and the presence of cardiovascular disease, cranial nerve disease, and endocrine disease was excluded.

The patient had a short, stocky build; she was $143-\mathrm{cm}$ tall and had kyphosis. She was undergoing treatment for diabetes mellitus. Hematological examination showed mild anemia. Electrocardiography (ECG) showed sinus 

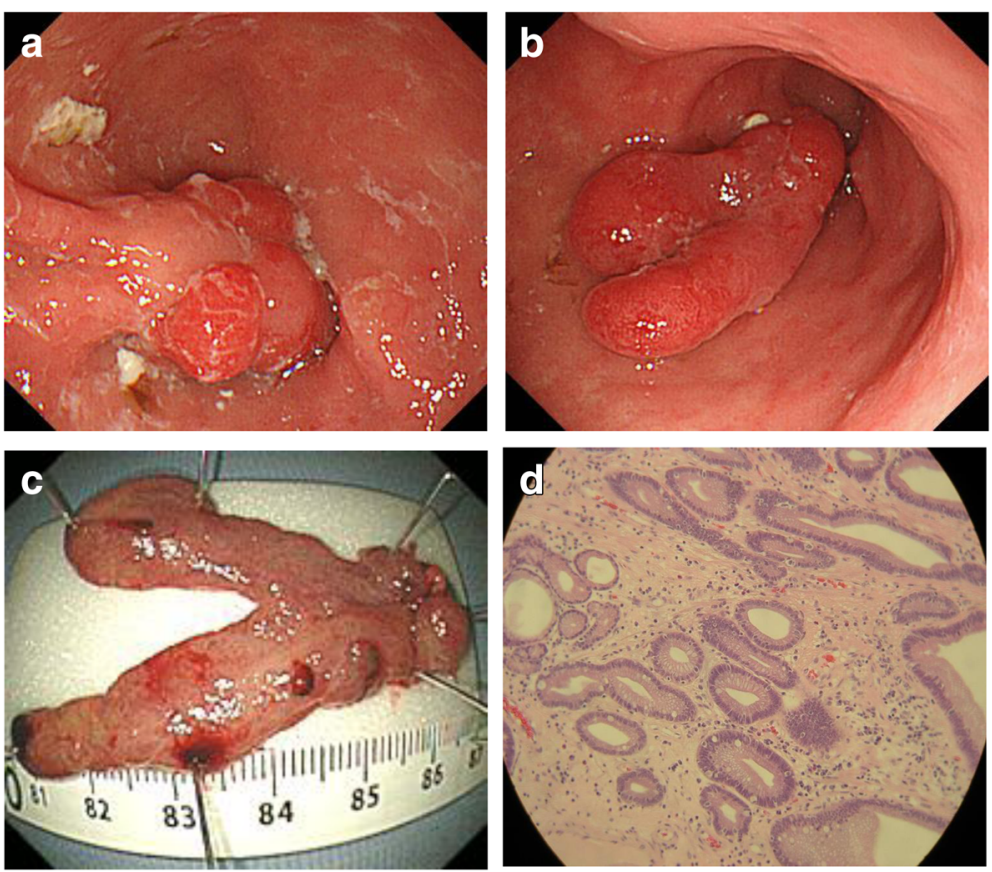

Fig. 1 Gastric polyp. Endoscopic examination revealed a a pedunculated polyp of the gastric antrum that fit into the pylorus and $\mathbf{b}$ a pedunculated polyp of the gastric antrum. c Polypectomy was performed using a detachable snare over the base of the stalk. $\mathbf{d}$ Pathological examination revealed a hyperplastic polyp with intestinal metaplasia without malignancy

rhythm with a heart rate of 64 beats/min and complete right bundle branch block. Esophagogastroduodenoscopy revealed a pedunculated polyp of the stomach antrum that fit into the pylorus (Fig. 1a, b). It showed a mixed type of hiatal hernia but no reflux esophagitis. In the upper gastrointestinal contrast study, the entire stomach had relocated to the thoracic cavity and the body of the stomach was located above the fundus, resulting in a so-called upside-down stomach (Fig. 2a). Contrast-enhanced computed tomography revealed that a large portion of the stomach, transverse colon, and part of the pancreas were present in the mediastinum (Fig. 2b). These structures compressed the left atrium.
The cause of syncope was suspected to be circulatory failure due to compression by the contents of the hiatal hernia. Therefore, we performed transthoracic echocardiography followed by a water pouring test using a nasogastric tube (Fig. 3). The test involved gradual instillation of saline into the stomach in the sitting position while performing blood pressure, ECG, and echocardiography monitoring. After $2000 \mathrm{ml}$ of saline had been instilled, the left atrium was markedly compressed and the area of the mitral annulus was reduced. No obvious ECG changes or syncope were observed, but a slight decrease in cardiac output was evident.
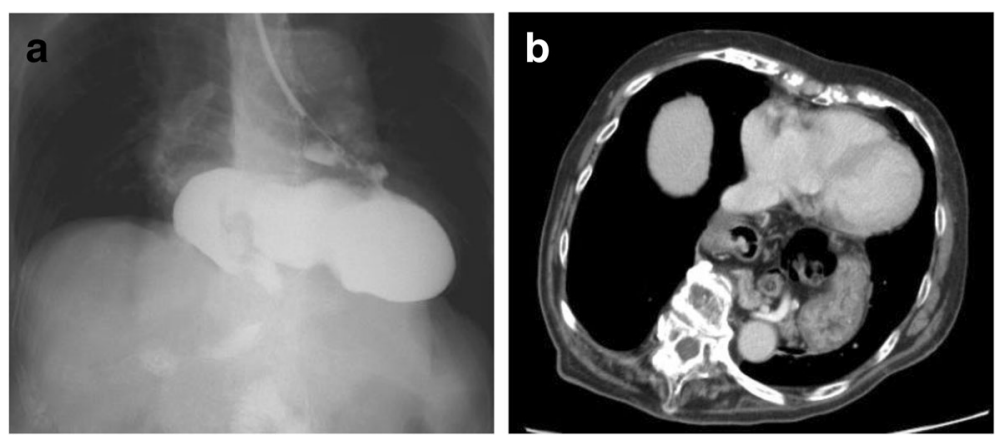

Fig. 2 Giant hiatal hernia. a An upper gastrointestinal contrast study showed an upside-down stomach. b Computed tomography revealed a giant hiatal hernia with the intrathoracic stomach located behind the heart and compressing the left atrium 


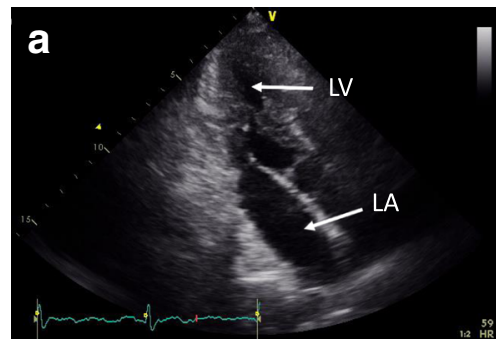

before water pouring test

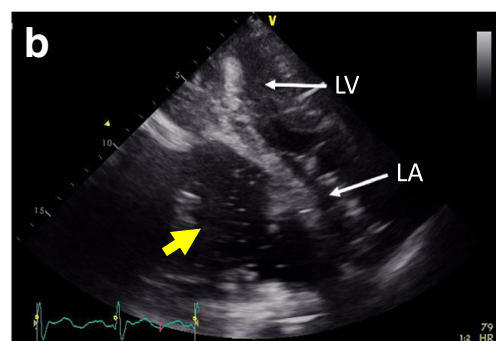

after water pouring test

Fig. 3 Transthoracic echocardiography. a Echocardiography before the water pouring test. b Echocardiography showed that after instillation of $2000 \mathrm{ml}$ of saline, the left atrium was markedly compressed and the area of the mitral annulus was reduced. The yellow arrow indicates saline in the stomach

Surgery with curative intent was performed (laparoscopic hiatal hernia repair and endoscopic gastric polypectomy). The esophageal hiatus was dilated to about $50 \mathrm{~mm}$, which revealed that a large portion of the stomach was present in the mediastinum. The hernia orifice was sutured closed after reduction of the hernia contents. Because this patient showed no evidence of reflux esophagitis, fundoplication was not performed. Finally, we performed polypectomy for removal of the gastric polyps by the usual method using a detachable snare. The postoperative course was good, and the patient was discharged uneventfully. She experienced no syncopal episodes for 5 months postoperatively.

\section{Conclusions}

The patient in this case developed syncope due to heart failure caused by a giant hiatal hernia. Syncope has a variety of causes, including cerebrovascular disease, arrhythmia, hypoglycemia, and autonomic nervous disorders. The vagal reflex is occasionally involved in swallowing. In the present case, we observed narrowing of the left atrium by cardiac ultrasonography. We found that content of hiatal hernia was the cause of the decreased cardiac output.

Including the present case, 10 cases in which a giant hiatal hernia was considered to be the cause of syncope were found in a search of PubMed/Medline from January 1995 to August 2016 (Table 1). The following search terms were used: hiatal hernia OR hiatus hernia AND syncope. Syncope caused by a hiatal hernia seems to be more prevalent in elderly women, with $80 \%$ female predominance and a mean age at onset of 79.4 years in women. The main diagnostic tools are computed tomography, ultrasonography, and chest X-ray. Chest X-ray should be considered because a hiatal hernia was diagnosed by X-ray in two reported cases. In most reports, the authors speculated that the syncope was caused by decreased cardiac output due to left atrial retraction. Importantly, the syncopal episodes frequently occurred after a meal. Six of seven patients underwent surgery. The surgical procedures involved hernia repair, reconstruction, and prevention of recurrence. In two reports, the patients underwent laparoscopic fundoplication. Laparoscopic surgery is useful for hiatal hernia repair [11]. In our case, the laparoscopic operation was

Table 1 Cases with syncope due to a hiatal hernia

\begin{tabular}{|c|c|c|c|c|c|c|}
\hline Case & First author (year) & Age & Sex & $\begin{array}{l}\text { Timing of syncope and accompanied } \\
\text { symptoms }\end{array}$ & Main inspection method & Treatment \\
\hline 1 & Akdemir (2001) & 70 & $\mathrm{~F}$ & 10-15 min after heavy meals & X-ray, CT & Nissen fundoplication \\
\hline 2 & Maekawa (2002) & 76 & $\mathrm{~F}$ & Immediately after meals & X-ray, CT & Nissen fundoplication \\
\hline 3 & Oishi (2004) & 76 & $\mathrm{~F}$ & Immediately after meals & US, CT & Not referred \\
\hline 4 & Ker (2004) & 64 & M & Severe chest pain After meals & $\mathrm{CT}$ & $\begin{array}{l}\text { Laparoscopic Nissen } \\
\text { fundoplication }\end{array}$ \\
\hline 5 & Karamitsos (2009) & 82 & $\mathrm{~F}$ & After meals & US, CT & Not referred \\
\hline 6 & Vanerio (2011) & 84 & $\mathrm{~F}$ & $\begin{array}{l}\text { After ingestion a carbonated } \\
\text { soft drink }\end{array}$ & $\mathrm{CT}$ & Nissen fundoplication \\
\hline 7 & Zwermann (2013) & 53 & $M$ & Chest discomfort & $\mathrm{CT}$ & Laparoscopic surgery \\
\hline 8 & Gunaruman (2013) & 86 & $\mathrm{~F}$ & Nothing & US, CT & Adjustment of diet \\
\hline 9 & Our case & 82 & $\mathrm{~F}$ & During and immediately after meals & US, X-ray, CT & Hiatal hernia radical operation \\
\hline
\end{tabular}


performed safely and effectively. No mortality occurred in any of these reports.

We also performed a water pouring test as described by Maekawa et al. [3]. The patient's vital signs, ECG, blood pressure, oxygen saturation, and consciousness level were monitored to ensure patient safety. The patient was sitting on the chair inserting nasogastric tube, which was placed in the stomach in our study. And we infused water in the stomach step by step observing monitors. We stopped the test when left atrial shrinkage and decreased cardiac output appeared before syncope. We believe that the water pouring test should be very carefully performed with strict monitoring.

Gastric polyps are present in 0.5 to $2.0 \%$ of the general population and are usually asymptomatic [12, 13]. Hyperplastic polyps are reportedly the most common type, followed by fundic gland polyps [12]. In the present case, the patient's gastric hyperplastic polyp prolapsed into the pyloric ring; this conceivably led to failure of gastric emptying, which in turn caused expansion of the stomach in the mediastinum and resultant syncope. We performed simultaneous endoscopic polypectomy and laparoscopic hiatal hernia repair. The current standard treatment strategy for gastric polyps causing obstructive symptoms is complete removal either endoscopically or surgically, followed by pathological analysis. To the best of our knowledge, this is the first report of cardiogenic syncope caused by a gastric polyp accompanied by a giant hiatal hernia.

Parikh et al. [13] analyzed 39 reports of gastric polyps that led to gastric outlet obstruction in the Englishlanguage literature. The median size of the endoscopically removed polyps was $3 \mathrm{~cm}$, and the maximum size was $8 \mathrm{~cm}$. The maximum size of all polyps, including those removed surgically, was $13 \mathrm{~cm}$. The gastric polyp in the present case was about $6 \mathrm{~cm}$. The failure of gastric emptying led to stagnation of the gastric contents, which is considered to have promoted gastric distension into the mediastinum. Such a situation is likely to cause syncope. Clinicians should recognize that a hiatal hernia is a risk factor for syncope.

In summary, compression of the heart by a large hiatal hernia resulted in dynamic circulatory changes and syncope. The patient's gastric polyp facilitated this phenomenon by inhibiting gastric emptying.

Abbreviation

ECG: Electrocardiography

\section{Funding}

This work was supported in part by Grants-in-Aid for Scientific Research from the Japan Society for the Promotion of Science (JSPS) (grant numbers 16K11339, 16K10450, 15H04932, 16K15606, 17K10534 0006, 17K16529 0007 and 17K14982 0001).

\section{Authors' contributions}

HS served as the first author of this manuscript. TM performed the operation and analyzed the data. HS, MS, and MS assisted with the operation. HK and TM determined the pathological diagnosis. HH, YK, TYoshida, TYokobori and HK discussed the significance of this manuscript. KK performed transthoracic echocardiography and advised performance of the water pouring test. All authors read and approved the final manuscript.

Ethics approval and consent to participate

Not applicable.

\section{Consent for publication}

The patient provided consent for publication of her case.

Competing interests

The authors declare that they have no competing interests.

\section{Publisher's Note}

Springer Nature remains neutral with regard to jurisdictional claims in published maps and institutional affiliations.

\section{Author details}

'Department of General Surgical Science, Gunma University Graduate School of Medicine, 3-39-22 Showa-machi, Maebashi, Gunma 371-8511, Japan.

${ }^{2}$ Department of Molecular Pharmacology and Oncology, Gunma University Graduate School of Medicine, 3-39-22 Showa-machi, Maebashi, Gunma 371-8511, Japan. ${ }^{3}$ Department of Clinical Laboratory Center, Gunma University Hospital, 3-39-22 Showa-machi, Maebashi, Gunma 371-8511, Japan.

Received: 23 August 2017 Accepted: 3 December 2017

Published online: 13 December 2017

References

1. Torres D, Parrinello G, Cardillo M, Pomilla M, Trapanese C, Michele B, et al. Large hiatal hernia at chest radiography in a woman with cardiorespiratory symptoms. Am J Emerg Med. 2012;30(9):2103-e1-3.

2. Hokamaki J, Kawano H, Miyamoto S, Sugiyama S, Fukushima R, Sakamoto T, et al. Dynamic electrocardiographic changes due to cardiac compression by a giant hiatal hernia. Internal Med (Tokyo, Japan). 2005;44(2):136-40.

3. Maekawa T, Suematsu M, Shimada T, Go M, Shimada T. Unusual swallow syncope caused by huge hiatal hernia. Internal Med (Tokyo, Japan). 2002;41(3): 199-201.

4. Oishi Y, Ishimoto T, Nagase N, Mori K, Fujimoto S, Hayashi S, et al. Syncope upon swallowing caused by an esophageal hiatal hernia compressing the left atrium: a case report. Echocardiography (Mount Kisco, NY). 2004;21(1):61-4.

5. Karamitsos TD, Arnold JR, Rider OJ, Francis JM, Ferrett C, Neubauer S, et al. Massive hiatus hernia impeding transoesophageal echocardiography in a patient with swallow-syncope syndrome. Hellenic Journal of Cardiology: HJC = Hellenike Kardiologike Epitheorese. 2009;50(3):216-7.

6. Zwermann L, Rittler P, Spelsberg F, Helck A, Pratschke S, Methe H. Syncope due to a massive upside-down stomach. J Am Coll Cardiol. 2013;61 (18):1925.

7. Gunaruwan P, Napthali K, Nair K. Unusual presentation of a common problem. BMJ Case Rep. 2013;2013. doi:10.1136/bcr-2012-007973.

8. Ker J, Van Beljon J. Diaphragmatic hernia mimicking an atrial mass: a twodimensional echocardiographic pitfall and a cause of postprandial syncope. Cardiovascular J of South Africa: official journal for Southern Africa Cardiac Society [and] South African Society of Cardiac Practitioners. 2004;15(4):182-3.

9. Akdemir I, Davutoglu V, Aktaran S. Giant hiatal hernia presenting with stable angina pectoris and syncope-a case report. Angiology. 2001;52(12):863-5.

10. Vanerio G. Syncope caused by huge hiatal hernia. Case Reports Cardiol. 2011;2011:560734

11. Fullum TM, Oyetunji TA, Ortega G, Tran DD, Woods IM, Obayomi-Davies O, Pessu O, Downing SR, Cornwell EE. Open versus laparoscopic hiatal hernia repair. JSLS. 2013;17(1):23-9.

12. Morais DJ, Yamanaka A, Zeitune JM, Andreollo NA. Gastric polyps: a retrospective analysis of 26,000 digestive endoscopies. Arq Gastroenterol. 2007:44(1):14-7.

13. Parikh M, Kelley B, Rendon G, Abraham B. Intermittent gastric outlet obstruction caused by a prolapsing antral gastric polyp. World J Gastrointestinal Oncol. 2010;2(5):242-6. 